\title{
A RELATION-THEORETIC EXPANSION PRINCIPLE
}

\author{
M. ImDad, W.M. AlfaqiH
}

Abstract. In this paper, we present a new and novel generalization of the expansion principle on a metric space endowed with a binary relation which, under universal relation, reduces to the well-known expansion principle due to Wang et al. [Math. Japon. 29, 4 (1984), 631-636]. Our findings possibly pave the way for another direction of relation-theoretic metrical fixed point results. We furnish an example to exhibit the utility of our results. Finally, we utilize one of our results to prove a fixed point theorem for cyclic expansive mappings.

2010 Mathematics Subject Classification: 47H10, 54H25.

Keywords: Expansive mappings, cyclical mappings, fixed point, binary relation.

\section{INTRODUCTION}

The advancement and the rich growth of fixed point theory have important theoretical and practical applications in almost all scientific fields of study. The development has been tremendous in yesteryears. Usually, fixed point theory uses to prove the existence and uniqueness of the solutions of huge variety of equations arising in theoretical and practical disciplines of Mathematics. The celebrated Banach contraction principle is one of the most efficient as well as powerful tools to study such problems. Due to enormous utility and applications, Banach contraction principle has been generalized in several ways and with the same spirit Wang et al. [32] initiated the study of expansion mappings in metric spaces wherein the authors defined expansion mapping and utilize the same to prove the following theorem:

Theorem 1. Let $(M, d)$ be a complete metric space and $g$ a self-mapping on $M$. If $g$ is surjective and satisfies

$$
d(g u, g v) \geq \lambda d(u, v)
$$

for all $u, v \in M$ with $\lambda>1$, then $g$ has a unique fixed point in $M$. 
M. Imdad, W.M. Alfaqih - A relation-theoretic expansion principle ...

Observe that the condition $\lambda>1$ is crucial, e. $g$. the function $g:[0, \infty) \rightarrow[0, \infty)$ given by $g u=2 u+e^{u}$ satisfies $d(g u, g v) \geq d(u, v)$ for all $u, v \in[0, \infty)$, and $g$ has no fixed point, where $d$ stands for the usual metric on $[0, \infty)$.

Theorem 1 has been extend and generalized in many directions (see [2, 14, 24, $30,12,1,9,20,11,22,23]$ and references cited therein).

In resent years, various results in fixed point theory were proved in metric spaces endowed with different types of binary relations (see [4, 3, 6, 7, 21, 25, 26, 27, 29, 31] and references cited therein). In this context, we employ an arbitrary binary relation to present a new generalization of Theorem 1.

In this paper, we extend the well known expansion principle to a metric space endowed with a binary relation. In this context, the expansion condition is relatively weaker than the usual condition as it is required to hold only on those elements which are related under the underlying relation rather than the whole space. Particularly, under the universal relation, our main result reduces to the well known expansion principle due to Wang et al. [32]. In the process, we require to introduce some new notions namely: orbitally $\mathcal{S}$-continuous and $\mathcal{S}$-precompleteness which are relatively weaker than their analogous conditions existing in the literature. Further, we furnish an example to show the utility of our results. Finally, we apply one of our results to prove a fixed point theorem for cyclic expansive mappings.

As usual $\mathbb{N}$ is the set of natural numbers while $\mathbb{N}_{0}=\{0\} \cup \mathbb{N}$. In the sequel, $M$ is a nonempty set, $g: M \rightarrow M$ a surjective map and $g_{r}^{-1}$ refers for a right inverse of $g$ under composition, i.e., $g \circ g_{r}^{-1}=I_{M}\left(I_{M}\right.$ is the identity map on $\left.M\right)$. For brevity, we write $g u$ instead of $g(u),\left\{u_{n}\right\} \rightarrow u$ whenever the sequence $\left\{u_{n}\right\}$ converges to $u$ and for all $n$ one means that for all $n \in \mathbb{N}_{0}$. A point $u \in M$ is said to be a fixed point of $g$ if $g u=u$ whereas Fix $(g)$ denotes the set of all such points. Let $u_{0} \in M$, a sequence $\left\{u_{n}\right\} \subseteq M$ which is defined by $u_{n+1}=g^{n} u_{0}=g u_{n}$, for all $n$, is called a Picard sequence based on $u_{0}$.

\section{RELATION THEORETIC NOTIONS AND AUXILIARY RESUltS}

In this section, we present some definitions and basic results which are needed in the sequel.

$A$ binary relation on $M$ is a non-empty subset $\mathcal{S}$ of $M \times M$. Trivially, $M \times M$ is always a binary relation on $M$ known as universal relation. For simplicity, we write $u \mathcal{S} v$ whenever $(u, v) \in \mathcal{S}$ and $u \mathcal{S}^{H} v$ whenever $u \mathcal{S} v$ and $u \neq v$. Observe that $\mathcal{S}^{H}$ is also a binary relation on $M$ such that $\mathcal{S}^{+} \subseteq \mathcal{S}$. The points $u$ and $v$ are said to be $\mathcal{S}$-comparable if $u \mathcal{S} v$ or $v \mathcal{S} u$, this is denoted by $[u, v] \in \mathcal{S}$. Throughout this work, $\mathcal{S}$ stands for a binary relation defined on $M, \mathcal{S}_{M}$ stands for the universal relation on $M$ and $M(g, \mathcal{S})=\{u \in M: u \mathcal{S} g u\}$. 
M. Imdad, W.M. Alfaqih - A relation-theoretic expansion principle ...

Definition 1. (see $[18,19,26])$ A binary relation $\mathcal{S}$ is said to be:

(i) amorphous if it is an arbitrary relation;

(ii) reflexive if for all $u \in M, u \mathcal{S} u$;

(iii) transitive if for any $u, v, z \in M, u \mathcal{S} v$ and $v \mathcal{S} z$ imply $u \mathcal{S} z$;

(iv) antisymmetric if for any $u, v \in M, u \mathcal{S} v$ and $v \mathcal{S} u$ imply $u=v$;

$(v)$ partial order if it is reflexive, transitive and antisymmetric;

(vi) complete, connected or dichotomous if $[u, v] \in \mathcal{S}$ for all $u, v \in M$.

Definition 2. [28] Let $M$ be a non-empty set, $E \subseteq M$ and $\mathcal{S}$ a binary relation on $M$. If for each $u, v \in E$, there exists $z \in M$ such that $u \mathcal{S} z$ and $v \mathcal{S} z$, then $E$ is called $\mathcal{S}$-directed.

Definition 3. [16] Let $M$ be a non-empty set and $u, v \in M$. A path of length $p$ $(p \in \mathbb{N})$ in $\mathcal{S}$ from $u$ to $v$ is a finite sequence $\left\{u_{0}, u_{1}, \ldots, u_{p}\right\} \subseteq M$ satisfying the following:

(i) $u_{0}=u$ and $u_{p}=v$;

(ii) $\left(u_{i}, u_{i+1}\right) \in \mathcal{S}$ for each $i \in\{0,1, \ldots, p-1\}$.

Observe that if $L$ is a path from $u$ to $v$ of length $p$, then $L$ involves $p+1$ elements of $M$, although they are not necessarily distinct.

Definition 4. [5] Let $M$ be a non-empty set and $E \subseteq M$. If for each $u, v \in E$ there exists a path in $\mathcal{S}$ from $u$ to $v$, then $E$ is said to be $\mathcal{S}$-connected.

Definition 5. [17] Let $\mathcal{S}$ a binary relation on a non-empty set $M$.

- $\mathcal{S}^{-1}=\left\{(u, v) \in M^{2}:(v, u) \in \mathcal{S}\right\}$ known as the inverse, transpose or dual relation of $\mathcal{S}$;

- $\mathcal{S}^{s}=\mathcal{S} \cup \mathcal{S}^{-1}$ is the symmetric closure of $\mathcal{S}$.

The following proposition immediate follows from the fact that $\mathcal{S} \subseteq \mathcal{S}^{s}$.

Proposition 1. Let $\mathcal{S}$ a be binary relation on a non-empty set $M$. If $M$ is $\mathcal{S}$ connected, then it is also $\mathcal{S}^{s}$-connected.

Definition 6. [4] Let $M$ be a non-empty set, $\mathcal{S}$ a binary relation on $M$ and $g$ : $M \rightarrow M$. Then $\mathcal{S}$ is called $g$-closed if $u \mathcal{S} v$ implies guS $\mathcal{S} v$ (for any $u, v \in M$ ). 
M. Imdad, W.M. Alfaqih - A relation-theoretic expansion principle ...

Definition 7. [5] Let $\mathcal{S}$ be a binary relation on a non-empty set $M$ and $\left\{u_{n}\right\} \subseteq M$. If $u_{n} \mathcal{S} u_{n+1}$ for all $n$, then $\left\{u_{n}\right\}$ is called $\mathcal{S}$-preserving sequence.

Definition 8. [10] Let $M$ be a non-empty set, $\mathcal{S}$ a binary relation on $M$ and $g$ : $M \rightarrow M$. A sequence $\left\{u_{n}\right\} \subseteq M$ is said to be $(g, \mathcal{S})$-Picard sequence if it is a Picard sequence with $u_{n} \mathcal{S} u_{n+1}$ (for all $n$ ).

In what follows we discuss certain types of continuity of a mapping. The first one of them is well known and often used in metric fixed point theory.

Definition 9. [8] Let $(M, d)$ be a metric space. A self-mapping $g: M \rightarrow M$ is said to be an orbitally continuous if for all $u, v \in M$ and any sequence $\left\{n_{i}\right\}$ of positive integers with $\left\{g^{n_{i}} u\right\} \rightarrow v$, we have $\left\{g g^{n_{i}} u\right\} \rightarrow g v$.

Observe that every continuous mapping is orbitally continuous.

Definition 10. [5] Let $(M, d)$ be a metric space, $\mathcal{S}$ a binary relation on $M$ and $u \in M$. A self-mapping $g: M \rightarrow M$ is said to be $\mathcal{S}$-continuous at $u$ if for any $\mathcal{S}$-preserving sequence $\left\{u_{n}\right\} \subseteq M$ such that $\left\{u_{n}\right\} \rightarrow u$, we have $\left\{g u_{n}\right\} \rightarrow$ gu. Furthermore, $g$ is called $\mathcal{S}$-continuous if it is $\mathcal{S}$-continuous at each point of $M$.

Remark 1. Every continuous mapping is $\mathcal{S}$-continuous, for any binary binary relation $\mathcal{S}$. Particularly, under the universal relation $\mathcal{S}_{M}$ the notion of $\mathcal{S}_{M}$-continuity coincides with usual continuity.

Inspired by the above types of continuity, we introduce the notion of orbital $\mathcal{S}$-continuity as follows:

Definition 11. Let $(M, d)$ be a metric space and $\mathcal{S}$ a binary relation on $M$. A self-mapping $g: M \rightarrow M$ is said to be an orbitally $\mathcal{S}$-continuous if for all $u, v \in M$ and any sequence $\left\{n_{i}\right\}$ of positive integers, we have

$$
\left\{g^{n_{i}} u\right\} \rightarrow v \text { and } g^{n_{i}} u \mathcal{S} g^{n_{i+1}} u \text { for all } i \in \mathbb{N} \text { imply }\left\{g g^{n_{i}} u\right\} \rightarrow g v .
$$

Remark 2. Every orbitally continuous mapping is orbitally $\mathcal{S}$-continuous, for any arbitrary binary relation $\mathcal{S}$. Especially, under the universal relation $\mathcal{S}_{M}$ the notion of orbital $\mathcal{S}_{M}$-continuity coincides with orbital continuity.

Remark 3. The following implications are obvious:

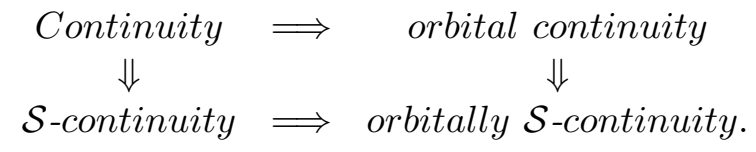

Definition 12. [29] Let $(M, d)$ be a metric space. A subset $E \subseteq M$ is said to be precomplete if each Cauchy sequence $\left\{u_{n}\right\} \subseteq E$ converges to some $u \in M$. 
M. Imdad, W.M. Alfaqih - A relation-theoretic expansion principle ...

Observe that every complete subset of $M$ is precomplete.

Definition 13. Let $\mathcal{S}$ be a binary relation on a non-empty set $M$ and $d$ a metric on $M$. A subset $E \subseteq M$ is said to be $\mathcal{S}$-precomplete if each $\mathcal{S}$-preserving Cauchy sequence $\left\{u_{n}\right\} \subseteq E$ converges to some $u \in M$.

Remark 4. Every precomplete subset of $M$ is $\mathcal{S}$-precomplete, for an arbitrary binary relation $\mathcal{S}$.

Observe that the converse of Remark 4 need not to be true in general. To substantiate the claim, we present the following example:

Example 1. Let $M=(0, \infty)$ equipped with the usual metric. Define a binary relation $\mathcal{S}$ on $M$ as follows:

$$
u \mathcal{S} v \Longleftrightarrow u \geq v \geq 1 \text { and } u, v \in \mathbb{Q} \text {. }
$$

Observe that $\left\{\frac{1}{n}\right\}$ is a Cauchy sequence which has no limit point in $M$ so that $M$ is not precomplete. Clearly, $M$ is $\mathcal{S}$-precomplete.

Definition 14. [4] Let $(M, d)$ be a metric space endowed with a binary relation $\mathcal{S}$. Then $\mathcal{S}$ is said to be $d$-self-closed if whenever $\left\{u_{n}\right\}$ is an $\mathcal{S}$-preserving sequence converging to $u$, there exists a subsequence $\left\{u_{n_{k}}\right\} \subseteq\left\{u_{n}\right\}$ such that $\left[u_{n_{k}}, u\right] \in \mathcal{S}$ for all $k \in \mathbb{N}_{0}$.

Lemma 2. If $g: M \rightarrow M$ is a surjective mapping, then it has $g_{r}^{-1}$.

Proof. Let $u \in M$ be an arbitrary point. Let $v_{u} \in M$ be any point such that $g v_{u}=u$. Define a mapping $G: M \rightarrow M$ by: $G u=v_{u}$ for all $u \in M$. Observe that, for all $u \in M$, we have $(g \circ G) u=g(G u)=g v_{u}=u$. Hence, $G=g_{r}^{-1}$.

Proposition 2. If $g$ is a surjective self-mapping on a non-empty set $M$, then Fix $\left(g_{r}^{-1}\right) \subseteq$ Fix $(g)$.

Proof. Lemma 2 ensures the existence of $g_{r}^{-1}$. Now, let $u \in F i x\left(g_{r}^{-1}\right)$. Then $g_{r}^{-1} u=$ $u$ which implies that $g \circ g_{r}^{-1} u=g u$ implying thereby $u=g u$ so that $u \in F i x(g)$. This shows that Fix $\left(g_{r}^{-1}\right) \subseteq$ Fix $(g)$.

Proposition 3. If $g$ is a bijective self-mapping on a non-empty set $M$, then Fix $(g)=$ Fix $\left(g^{-1}\right)$.

Proof. As $g$ is bijective mapping, then $g^{-1}$ exists and is also bijective. In view of Proposition 2, we have Fix $\left(g^{-1}\right) \subseteq$ Fix $(g)$. Now, let $u \in F i x(g)$. Then we have $g u=u$ which implies that $g^{-1} u=u$ yielding there by Fix $(g) \subseteq$ Fix $\left(g^{-1}\right)$. Hence, $\operatorname{Fix}(g)=\operatorname{Fix}\left(g^{-1}\right)$. 
M. Imdad, W.M. Alfaqih - A relation-theoretic expansion principle ...

The following proposition immediate owing to the symmetricity of the metric $d$.

Proposition 4. If $g$ is a self-mapping on a metric space $(M, d)$, then for each $\lambda>1$, the following are equivalent:

(a) $d(g u, g v) \geq \lambda d(u, v)$ for all $u, v \in M$ such that $(u, v) \in \mathcal{S}$;

(b) $d(g u, g v) \geq \lambda d(u, v)$ for all $u, v \in M$ such that $[u, v] \in \mathcal{S}$.

\section{Fixed POINT RESUlts}

Before giving our results, let us highlight the fact that we utilize the " $\mathcal{S}^{\text {H}}$-precompleteness of $g M$ " in our results which is relatively weaker than the following conditions utilized by earlier authors:

1. $g M$ is $\mathcal{S}$-complete;

2. $g M$ is precomplete;

3. $M$ or $g M$ is complete;

4. there exists a complete subset $H \subseteq M$ such that $g M \subseteq H \subseteq M$;

5. $M$ is complete and $g M$ is closed.

Observe that if any one of these five conditions holds, then $g M$ is $\mathcal{S}^{H}$-precomplete. Also, we use orbital $\mathcal{S}^{H}$-continuity which is relatively weaker as compare to orbital continuity as well as $\mathcal{S}$-continuity.

Now, we are equipped to prove our main results starting with the following existence result:

Theorem 3. Let $(M, d)$ be a metric space endowed with a binary relation $\mathcal{S}$ and $g$ a surjective self-mapping on $M$. Assume that the following conditions are satisfied:

(a) $M\left(g_{r}^{-1}, \mathcal{S}\right)$ is non-empty;

(b) $\mathcal{S}$ is $g_{r}^{-1}$-closed;

(c) $g M$ is $\mathcal{S}^{H}$-precomplete;

(d) $g$ is orbitally $\mathcal{S}^{H}$-continuous;

(e) there exists $\lambda>1$ such that

$$
d(g u, g v) \geq \lambda d(u, v) \text { for all } u, v \in M \text { such that } u \mathcal{S}^{H} v .
$$


Then $g$ has a fixed point. Indeed, if $\left\{u_{n}\right\}$ is any $\left(g_{r}^{-1}, \mathcal{S}\right)$-Picard sequence, then either $\left\{u_{n}\right\}$ contains a fixed point of $g$ or $\left\{u_{n}\right\}$ converges to a fixed point of $g$.

Proof. In view of Lemma $2, g$ has $g_{r}^{-1}$. Now, let $u, v \in M$ be arbitrary points such that $u \mathcal{S}^{H} v$ and let $z=g_{r}^{-1} u$ and $w=g_{r}^{-1} v$ (obviously, $z \mathcal{S}^{H} w$ as $\mathcal{S}$ is $g_{r}^{-1}$-closed and $u \neq v)$. Now, applying condition $(e)$, with $z$ and $w$, we have

$$
d(g z, g w) \geq \lambda d(z, w)
$$

as $g z=g g_{r}^{-1} u=u$ and $g w=g g_{r}^{-1} v=v$, we get

$$
d\left(g_{r}^{-1} u, g_{r}^{-1} v\right) \leq \frac{1}{\lambda} d(u, v)
$$

Since $u$ and $v$ were arbitrary, therefore (1) holds for all $u, v$ such that $u \mathcal{S}^{\sharp} v$. Observe that hypothesis $(a)$ guarantees the existence of a point $u_{0} \in M$ such that $u_{0} \mathcal{S} g_{r}^{-1} u_{0}$. Let $u_{1} \in M$ such that $u_{1}=g_{r}^{-1} u_{0}$. Hence, we have $u_{0} \mathcal{S} u_{1}$ and as $\mathcal{S}$ is $g_{r}^{-1}$-closed, we have $g_{r}^{-1} u_{0} \mathcal{S} g_{r}^{-1} u_{1}$. Similarly, there exists $u_{2} \in M$ such that $u_{2}=g_{r}^{-1} u_{1}$ and $u_{1} \mathcal{S} u_{2}$. Thus, inductively, we can construct a sequence $\left\{u_{n}\right\} \subseteq M$ such that $u_{n+1}=g_{r}^{-1} u_{n}$ and $u_{n} \mathcal{S} u_{n+1}$ for all $n$. If there exists $n_{0} \in \mathbb{N}_{0}$ such that $u_{n_{0}}=g_{r}^{-1} u_{n_{0}}$, then $g u_{n_{0}}=u_{n_{0}}$ and the result is established. Assume that $u_{n+1} \neq u_{n}$ for all $n$. Then $\left\{u_{n}\right\}$ is $\mathcal{S}^{H}$-preserving sequence. On using (1), for all $n$, we have

$$
d\left(g_{r}^{-1} u_{n+1}, g_{r}^{-1} u_{n}\right) \leq \frac{1}{\lambda} d\left(u_{n+1}, u_{n}\right),
$$

which by induction yields that

$$
d\left(u_{n+2}, u_{n+1}\right) \leq\left(\frac{1}{\lambda}\right)^{n+1} d\left(u_{1}, u_{0}\right) \text { for all } n .
$$

Let $n, m \in \mathbb{N}_{0}$ such that $n<m$.

Now, on using triangle inequality and (2), we have

$$
\begin{aligned}
d\left(u_{n}, u_{m}\right) & \leq d\left(u_{n}, u_{n+1}\right)+d\left(u_{n+1}, u_{n+2}\right)+\ldots+d\left(u_{m-1}, u_{m}\right) \\
& \leq\left(\left(\frac{1}{\lambda}\right)^{n}+\left(\frac{1}{\lambda}\right)^{n+1}+\ldots+\left(\frac{1}{\lambda}\right)^{m-1}\right) d\left(u_{0}, u_{1}\right) \\
& =\left(\frac{1}{\lambda}\right)^{n}\left(\sum_{i=0}^{m-n-1}\left(\frac{1}{\lambda}\right)^{i}\right) d\left(u_{0}, u_{1}\right) \\
& =\left(\frac{1}{\lambda}\right)^{n}\left(\frac{1-\left(\frac{1}{\lambda}\right)^{m-n}}{1-\frac{1}{\lambda}}\right) d\left(u_{0}, u_{1}\right) \\
& <\left(\frac{1}{\lambda}\right)^{n}\left(\frac{1}{1-\frac{1}{\lambda}}\right) d\left(u_{0}, u_{1}\right) \rightarrow 0 \text { as } n \rightarrow \infty
\end{aligned}
$$


M. Imdad, W.M. Alfaqih - A relation-theoretic expansion principle ...

which implies that $\left\{u_{n}\right\}$ is a Cauchy sequence. Thus, $\left\{u_{n}\right\}_{n \geq 1} \subseteq g M$ is a Cauchy $\mathcal{S}^{H}$-preserving sequence. Hence, there exists $u \in M$ such that $\left\{u_{n}\right\} \rightarrow u$ (due to condition $(c))$. Observe that $u_{n}=g^{n} u_{0}$ for all $n \in \mathbb{N}$ so that $\left\{g^{n} u_{0}\right\} \rightarrow u$. Moreover, $g^{n} u_{0} \mathcal{S}^{H} g^{n+1} u_{0}$ for all $n \in \mathbb{N}$. Since $g$ is orbitally $\mathcal{S}^{H}$-continuous, we get $\left\{u_{n+1}=g g^{n} u_{0}\right\}_{n \geq 1} \rightarrow g u$. Owing to the uniqueness of the limit, we obtain $g u=u$, i.e., $u$ is a fixed point of $g$. This concludes the proof.

Next, we use $d$-self-closedness to prove an analog of Theorem 3 wherein the continuity assumption of $g$ is avoided.

Theorem 4. Conclusions of Theorem 3 remain true if condition $(d)$ is replaced by the following:

$\left(d^{\prime}\right) \mathcal{S}^{H}$ is $d$-self-closed.

Proof. Following the proof of Theorem 3, we have $\left\{u_{n}\right\} \rightarrow u$. Now, we are required to prove that $g u=u$.

Since $\left\{u_{n}\right\}$ is $\mathcal{S}^{\text {H}}$-preserving and $\left\{u_{n}\right\} \rightarrow u$ and $\mathcal{S}^{\text {H }}$ is $d$-self-closed, therefore there exists a subsequence $\left\{u_{n_{k}}\right\}$ of $\left\{u_{n}\right\}$ such that

$$
\left[u_{n_{k}}, u\right] \in \mathcal{S}^{H} \text { for all } k \in \mathbb{N}_{0} .
$$

Using (1), (3) and Proposition 4, we obtain

$$
d\left(u_{n_{k}+1}, g_{r}^{-1} u\right)=d\left(g_{r}^{-1} u_{n_{k}}, g_{r}^{-1} u\right) \leq\left(\frac{1}{\lambda}\right) d\left(u_{n_{k}}, u\right)
$$

which on letting $k \rightarrow \infty$ gives rise $\left\{u_{n_{k}}\right\} \rightarrow g_{r}^{-1} u$. Again, owing to the uniqueness of the limit, we obtain $u=g_{r}^{-1} u$, which in turn implies that $g u=u$, i.e., $u$ is a fixed point of $g$.

The following result describes a set of sufficient conditions to ensure the uniqueness of the fixed point of $g$ which runs as follows:

Theorem 5. If in addition to the hypotheses of Theorem 3(or Theorem 4), we assume that, at least, one of the following conditions are fulfilled:

(i) Fix $(g)$ is $\mathcal{S}^{s}$-connected;

(ii) $g$ is bijective and for each $u, v \in F i x(g)$, there exists $z \in M$ such that $z$ is $\mathcal{S}$-comparable to $u$ and $v$ (at the same time).

Then $g$ has a unique fixed point. 
M. Imdad, W.M. Alfaqih - A relation-theoretic expansion principle ...

Proof. Assume that $(i)$ holds. In view of Theorem 3 (or Theorem 4), Fix $(g)$ is non-empty. Now, let $u, v \in F i x(g)$, then we are done if we show that $u=v$. Since Fix $(g)$ is $\mathcal{S}^{s}$-connected, therefore there exists a path of some finite length $p$ in $\mathcal{S}^{s}$ [say $\left.\left\{u_{0}, u_{1}, \ldots, u_{p}\right\} \subseteq F i x(g)\right]$ from $u$ to $v$ so that

$$
u_{0}=u, u_{p}=v \text { and }\left[u_{i}, u_{i+1}\right] \in \mathcal{S} \text { for each } i,(0 \leq i \leq p-1) .
$$

Since $u_{i} \in F i x(g)$, therefore $g u_{i}=u_{i}$ for each $i \in\{0,1, \ldots, p\}$. Hence, on using $(d)$, we obtain

$$
d\left(u_{i}, u_{i+1}\right)=d\left(g u_{i}, g u_{i+1}\right) \geq \lambda d\left(u_{i}, u_{i+1}\right) \text { for each } i,(0 \leq i \leq p),
$$

which implies that $d\left(u_{i}, u_{i+1}\right)=0$ for each $i \in\{0,1, \ldots, p\}$ yielding thereby $u=v$. Hence, $g$ has a unique fixed point.

Next, suppose that (ii) holds. Let $u, v \in F i x(g)$, by our assumption, there exists $z_{0} \in X$ such that $\left[u, z_{0}\right] \in \mathcal{S}$ and $\left[v, z_{0}\right] \in \mathcal{S}$. Let $\left\{z_{n}\right\}$ be a Picard sequence under $g^{-1}$ based on $z_{0}$, i.e., $z_{n+1}=g^{-1} z_{n}$ for all $n$. Now, we show that $u=v$ by proving that $\left\{z_{n}\right\} \rightarrow u$ and $\left\{z_{n}\right\} \rightarrow v$.

As $\left[u, z_{0}\right] \in \mathcal{S}$, we assume that $u \mathcal{S} z_{0}$ (the case $z_{0} \mathcal{S} u$ is similar). As $\mathcal{S}$ is $g^{-1}$-closed (in view of condition (b)), we have $u \mathcal{S} z_{n}$ for all $n$. If $u=z_{n_{0}}$ for some $n_{0} \in \mathbb{N}_{0}$, then $u=z_{n}$ for all $n \geq n_{0}$ so that $\left\{z_{n}\right\} \rightarrow u$. Assume that $u \neq z_{n}$ for all $n$. Then we have $u \mathcal{S}^{\dagger} z_{n}$ for all $n$. Setting $u=u$ and $v=z_{n-1}$ in inequality (1), we have

$$
d\left(u, z_{n}\right)=d\left(g^{-1} u, g^{-1} z_{n-1}\right) \leq \frac{1}{\lambda} d\left(u, z_{n-1}\right),
$$

(for all $n$ ) so that inductively, we have

$$
d\left(u, z_{n}\right) \leq\left(\frac{1}{\lambda}\right)^{n} d\left(u, z_{0}\right) \rightarrow 0, \text { as } n \rightarrow \infty .
$$

Thus, $\left\{z_{n}\right\} \rightarrow u$. Similarly, we can show that $\left\{z_{n}\right\} \rightarrow v$ which concludes the proof.

Now, we consider some special cases wherein our results deduce the following results of the existing literature.

- Under the universal relation $\mathcal{S}_{M}$, Theorem 5 reduces to Theorem 1.

- On setting $\mathcal{S}=\preceq$, the partial order relation, in Theorem 5, we obtain Corollary 3.4 of Karapnar et al. [13].

Corollary 6. Conclusions of Theorem 5 remain true if the $\mathcal{S}^{s}$-connectedness of Fix $(g)$ is replaced by any one of the following:

(i) Fix $(g)$ is $\mathcal{S}$-connected; 
(ii) Fix $(g)$ is $\mathcal{S}^{s}$-directed;

(iii) $\mathcal{S}$ is complete on Fix $(g)$.

Proof. Assume that $(i)$ holds, then the proof is accomplished in view of Proposition 1 and part $(i)$ of Theorem 5 .

If $(i i)$ holds, then for each $u, v \in F i x(g)$, there exists $z \in F i x(g)$ such that $u \mathcal{S} z$ and $v \mathcal{S} z$, i.e, $[u, z] \in \mathcal{S}^{s}$ and $[v, z] \in \mathcal{S}^{s}$ so that $\{u, z, v\} \subseteq F i x(g)$ is a path of length 2 from $u$ to $v$ in $\mathcal{S}^{s}$. Hence, Theorem 5 part $(i)$ gives rise to the conclusion.

Finally, assume that (iii) holds, then for each $u, v \in F i x(g),[u, v] \in \mathcal{S}$ so that $\{u, v\} \subseteq F i x(g)$ is a path of length 1 in $\mathcal{S}^{s}$ from $u$ to $v$. Hence, Theorem 5 part $(i)$ again gives the conclusion.

\section{EXAMPLE}

In this section, we present an example to exhibit that our results are genuine extensions of several earlier results especially due to Wang et al. [32] and Karapnar et al. $[13]$.

Example 2. Let $M=(0, \infty)$ equipped with the usual metric. Define a binary relation $\mathcal{S}$ on $M$ as follows:

$$
u \mathcal{S} v \Longleftrightarrow u \geq v \geq 1 \text { and } u, v \in \mathbb{Q} .
$$

Define a mapping $g: M \rightarrow M$ by:

$$
g u= \begin{cases}\frac{u}{2}, & \text { if } 0<u \leq 1 \\ 2 u-\frac{3}{2}, & \text { if } 1 \leq u<\infty .\end{cases}
$$

Observe that $g$ is surjective, orbitally $\mathcal{S}^{+}$-continuous and $g_{r}^{-1}$ is given by:

$$
g_{r}^{-1} u= \begin{cases}2 u, & \text { if } 0<u \leq \frac{1}{2} \\ \frac{1}{2} u+\frac{3}{4}, & \text { if } \frac{1}{2} \leq u<\infty\end{cases}
$$

Now, clearly $\frac{3}{2} \in M\left(g_{r}^{-1}, \mathcal{S}\right), \mathcal{S}$ is $g_{r}^{-1}$-closed and $g M$ is $\mathcal{S}^{H}$-precomplete. Also, for all $u, v \in M$ with $u \mathcal{S}^{H} v$, we have

$$
d(g u, g v)=\left|\left(2 u-\frac{3}{2}\right)-\left(2 v-\frac{3}{2}\right)\right|=2|u-v|>\frac{3}{2}|u-v|=\frac{3}{2} d(u, v),
$$

i.e., $g$ satisfies the expansion condition $(e)$ with $\lambda=\frac{3}{2}$. Thus, all the hypotheses of Theorem 3 are satisfied. Hence, $g$ has a fixed point. Furthermore, notice that Fix $(g)=\left\{\frac{3}{2}\right\}$ is $\mathcal{S}^{s}$-connected. Thus, Theorem 5 is applicable in the context of this 
M. Imdad, W.M. Alfaqih - A relation-theoretic expansion principle ...

example. Observe that $g$ has a unique fixed point (namely $u=\frac{3}{2}$ ).

Here it can be pointed out that in the context of the present example Theorem 1 is not applicable. Since $M$ is incomplete space as well as for all $u, v \in(0,1)$, we have

$$
\frac{1}{2}|u-v|=d(g u, g v)<d(u, v)=|u-v| .
$$

Furthermore, the binary relation $\mathcal{S}$ given in this example is not a partial order on M. Thus, Corollary 3.4 due to Karapnar et al. [13] cannot be used in this case. These substantiate the utility of our results over corresponding noted results. Thus, in all, we have extended all the related results.

\section{FiXed POINT RESUlts FOR CYCLICAL EXPANSION MAPPINGS}

By considering a cyclical contractive condition, Kirk et al. [15] obtained a new extension of the celebrated Banach contraction principle as given in the following theorem:

Theorem 7. Let $E$ and $H$ be two non-empty closed subsets of a complete metric space $(M, d)$. Assume that $g$ is a self-mapping on $M$ satisfying the following:

(a) $g(E) \subseteq H, g(H) \subseteq E$;

(b) there exists $\lambda \in(0,1)$ such that

$$
d(g u, g v) \leq \lambda d(u, v) \forall u \in E, v \in H .
$$

Then $g$ has a unique fixed point in $E \cap H$.

In this section, we apply Theorem 4 to prove an analog theorem of Theorem 7 for expansive mappings which runs as follows:

Theorem 8. Let $E$ and $H$ be two non-empty closed subsets of a complete metric space $(M, d)$ such that $M=E \cup H$. Assume that $g$ is a surjective self-mapping on $M$ satisfying the following:

(a) $g_{r}^{-1}(E) \subseteq H, g_{r}^{-1}(H) \subseteq E$;

(b) there exists $\lambda>1$ such that

$$
d(g u, g v) \geq \lambda d(u, v) \forall u \in E, v \in H .
$$

Then $g$ has a fixed point. 
Proof. Define a binary relation $\mathcal{S}$ as follows:

$$
u \mathcal{S} v \Longleftrightarrow(u, v) \in(E \times H) \cup(H \times E) .
$$

We claim that $\mathcal{S}^{+}$is $d$-self-closed. To prove this claim, let $\left\{u_{n}\right\} \subseteq M$ be an $\mathcal{S}^{H_{-}}$ preserving sequence which converges to some $u \in M$. Observe that $u \neq u_{n}$ for all $n$. Let $\mathcal{P}=\left\{n \in \mathbb{N}_{0}:\left(u_{n}, u_{n+1}\right) \in E \times H\right\}$ and $\mathcal{P}^{*}=\left\{n \in \mathbb{N}_{0}:\left(u_{n}, u_{n+1}\right) \in H \times E\right\}$. Observe that $\mathcal{P} \cup \mathcal{P}^{*}=\mathbb{N}_{0}$ so that, at least, one of these sets is infinite. Assume that $\mathcal{P}$ is infinite. Then it can be written as a strictly increasing sequence of ranks: $\{n(i): i \geq 0\}$, where $i \longmapsto n(i)$ is strictly increasing so that $\lim _{i \rightarrow \infty} n(i)=\infty$.

Let $m(i)=n(i)+1$ for all $i \in \mathbb{N}_{0}$. Then $\{m(i)\}_{i \geq 0}$ is also strictly increasing sequence of ranks: $\{m(i): i \geq 0\}$ such that $\lim _{i \rightarrow \infty} m(i)=\infty$.

Notice that $\left\{u_{n(i)}\right\}$ and $\left\{u_{m(i)}\right\}$ are two subsequences of $\left\{u_{n}\right\}$ having the following properties:

(i) $\left\{u_{n(i)}\right\} \rightarrow u$ and $\left\{u_{m(i)}\right\} \rightarrow u$;

(ii) $u_{n(i)} \neq u$ and $u_{m(i)} \neq u$ for all $i \in \mathbb{N}_{0}$;

(iii) $u_{n(i)} \in E$ and $u_{m(i)} \in H$, for all $i \in \mathbb{N}_{0}$.

Now, as $u \in M$, we must have either $u \in E$ or $u \in H$. If $u \in E$, then we have $\left(u_{m(i)}, u\right) \in H \times E$ so that $u_{m(i)} \mathcal{S}^{H} u$ for all $i \in \mathbb{N}_{0}$. On the other hand, assume that $u \in H$, then we have $\left(u_{n(i)}, u\right) \in E \times H$ so that $u_{n(i)} \mathcal{S}^{H} u$ for all $i \in \mathbb{N}_{0}$. Hence, in any case, we get a subsequence of $\left\{u_{n}\right\}$ satisfying Definition 14. The proof is similar in case $\mathcal{P}^{*}$ is infinite. Therefore, the claim is established.

Next, from condition $(a)$, we have

$$
(u, v) \in \mathcal{S} \Longrightarrow\left(g_{r}^{-1} u, g_{r}^{-1} v\right) \in \mathcal{S},
$$

for each $u, v \in M$. Thus, $\mathcal{S}$ is $g_{r}^{-1}$-closed. Moreover, as $E$ is nonempty, there exists $u_{0} \in E$. On using condition $(a)$ we have $g_{r}^{-1} u_{0} \in H$ so that $u_{0} \mathcal{S} g_{r}^{-1} u_{0}$. Thus, all the hypotheses of Theorem 4 are fulfilled. Hence, $g$ has a fixed point.

\section{REFERENCES}

[1] C. T. Aage and J. N. Salunke. Some fixed point theorems for expansion onto mappings on cone metric spaces. Acta Mathematica Sinica, 27(6):1101-1106, 2011.

[2] B. Ahmad, M. Ashraf, and B. Rhoades. Fixed point theorems for expansive mappings in d-metric spaces. Indian Journal of Pure \& Applied Mathematics, 32(10):1513-1518, 2001. 
M. Imdad, W.M. Alfaqih - A relation-theoretic expansion principle ...

[3] M. Ahmadullah, M. Imdad, and R. Gubran. Relation-theoretic metrical fixed point theorems under nonlinear contractions. Accepted for publication in Fixed Point Theory, 2017.

[4] A. Alam and M. Imdad. Relation-theoretic contraction principle. Journal of Fixed Point Theory and Applications, 17(4):693-702, 2015.

[5] A. Alam and M. Imdad. Relation-theoretic metrical coincidence theorems. Under proses in Filomat, arXiv:1603.09159 [math.FA], 2017.

[6] W. M. Alfaqih, R. Gubran, and M. Imdad. Coincidence and common fixed point results under generalized $(\mathcal{A}, \mathcal{S})_{f}$-contraction. Submitted, 2017.

[7] H. Ben-El-Mechaiekh. The ran-reurings fixed point theorem without partial order: A simple proof. Journal of Fixed Point Theory and Applications, 1(16):373$383,2014$.

[8] L. B. Ciric. On contraction type mappings. Math. Balkanica, (1):52-57, 1971.

[9] J. Grnicki. Fixed point theorems for $\mathcal{F}$-expanding mappings. Fixed Point Theory and Applications, 2017:9:1-10, 2017.

[10] M. Imdad, Q. Khan, W. M. Alfaqih, and R. Gubran. A relation theoretic $(F, \mathcal{R})$ contraction principle with applications to matrix equations. To appear in Bulletin of Mathematical Analysis and Applications, 2018.

[11] M. Imdad and T. I. Khan. Fixed point theorems for some expansive mapping via implicit relations. In Nonlinear Analysis Forum, volume 9, pages 209-218, 2004.

[12] S. Kang. Fixed points for expansion mappings. Math. Japon, 38(4):713-717, 1993.

[13] E. Karapnar, P. Shahi, J. Kaur, and S. S. Bhatia. Generalized ( $\xi, \alpha)$-expansive mappings and related fixed-point theorems. Journal of Inequalities and Applications, 2014(22):1-13, 2014.

[14] M. A. Khan, M. S. Khan, and S. Sessa. Some theorems on expansion mappings and their fixed points. Demonstratio Math, 19(3), 1986.

[15] W. Kirk, P. Srinivasan, and P. Veeramani. Fixed points for mappings satisfying cyclical contractive conditions. Fixed point theory, 4(1):79-89, 2003.

[16] B. Kolman, R. C. Busby, and S. Ross. Discrete mathematical structures. In Third Edition. PHI Pvt. Ltd., New Delhi, 2000.

[17] S. Lipschutz. Schaum's outline of theory and problems of set theory and related topics. 1964.

[18] S. Lipschutz. Theory and problems of set theory and related topics. Technical report, 1964.

[19] R. Maddux. Relation algebras: Studies in logic and the foundations of mathematics, vol. 150, 2006. 
M. Imdad, W.M. Alfaqih - A relation-theoretic expansion principle ...

[20] Z. Mustafa, F. Awawdeh, and W. Shatanawi. Fixed point theorem for expansive mappings in g-metric spaces. Int. J. Contemp. Math. Sci, 5(50):2463-2472, 2010.

[21] J. J. Nieto and R. Rodríguez-López. Contractive mapping theorems in partially ordered sets and applications to ordinary differential equations. Order, 22(3):223-239, 2005.

[22] V. Popa. A general fixed point theorem for implicit cyclic multi-valued contraction mappings. In Annales Mathematicae Silesianae, volume 29, pages 119-129, 2015 .

[23] V. Popa and A. Patriciu. A fixed point theorem for cyclic mappings satisfying an implicit relation on partial metric spaces. Acta Universitatis Apulensis, (47):49-60, 2016.

[24] S. Radenović, T. Došenović, T. A. Lampert, and Z. Golubovíc. A note on some recent fixed point results for cyclic contractions in b-metric spaces and an application to integral equations. Applied Mathematics and Computation, 273:155-164, 2016.

[25] A. C. Ran and M. C. Reurings. A fixed point theorem in partially ordered sets and some applications to matrix equations. Proceedings of the American Mathematical Society, pages 1435-1443, 2004.

[26] A.-F. Roldán-López-de Hierro and N. Shahzad. Common fixed point theorems under $(\mathcal{R}, \mathcal{S})$-contractivity conditions. Fixed Point Theory and Applications, 2016(1):1-25, 2016.

[27] B. Samet and M. Turinici. Fixed point theorems on a metric space endowed with an arbitrary binary relation and applications. Communications in Mathematical Analysis, 13(2):82-97, 2012.

[28] B. Samet, C. Vetro, and P. Vetro. Fixed point theorems for $\alpha-\psi$-contractive type mappings. Nonlinear Analysis: Theory, Methods and Applications, 75(4):2154-2165, 2012.

[29] N. Shahzad, A. F. R. L. de Hierro, and F. Khojasteh. Some new fixed point theorems under $(\mathcal{A}, \mathcal{S})$-contractivity conditions. Revista de la Real Academia de Ciencias Exactas, Físicas y Naturales. Serie A. Matemáticas, pages 1-18, 2016.

[30] T. Taniguchi. Common fixed point theorems on expansion type mappings on complete metric spaces. Math. Japon., 34:139-142, 1989.

[31] M. Turinici. Contractive maps in locally transitive relational metric spaces. The Scientific World Journal, 2014, 2014.

[32] S. Z. Wang, B. Y. Li, Z. M. Gao, and K. Iski. Some fixed point theorems on expansion mappings. Math. Japon., 29(4):631-636, 1984.

Mohammad Imdad

Department of Mathematics, Faculty of Science, 
Aligarh Muslim University,

Aligarh-202002, India

email: mhimdad@yahoo.co.in

Waleed M. Alfaqih

Department of Mathematics, Faculty of Science,

Aligarh Muslim University,

Aligarh-202002, India

email: waleedmohd2016@gmail.com 